\title{
Crowned Dens Syndrome Incidentally Diagnosed After Investigation of Cervical Spine Injury With Incomplete Quadriplegia: A Case Report and Review of the Literature
}

\author{
Vasileios S. Nikolaou $^{\mathrm{a}, \mathrm{c}}$, Demetrios Chytas ${ }^{\mathrm{a}}$, Demitrios Korres ${ }^{\mathrm{b}}$, Nicolas Efstathopoulos ${ }^{\mathrm{a}}$
}

\begin{abstract}
Crowned dens syndrome (CDS) is a rare clinical entity characterized by acute neck pain due to calcification around the odontoid process of the axis in "crown-like" configuration. The disease can cause neurological symptoms, due to spinal cord compression but also it may lead to chronic neck pain or be asymptomatic. We present a case of a 73 -year-old patient who, after a fall of a height of approximately $2 \mathrm{~m}$, sustained a cervical spine injury and his clinical examination revealed an incomplete quadriplegia. Surprisingly, the only finding after the diagnostic imaging, including radiographs, CT scan and MRI, was periodontoid calcification, indicative of CDS. After a few days of hospital admission, a full neurological recovery was spontaneously achieved and the patient, after a 6-week use of cervical orthosis, was free of pain. We perform a brief review of the literature to shed light onto this rare clinical entity.
\end{abstract}

Keywords: Crowned dens syndrome; Odontoid; Symptoms; Axis; Treatment

\section{Introduction}

Crowned dens syndrome (CDS), also known as acute pseudogout of the cervical spine, characterized by calcifications around the odontoid process of the axis in a "crown-like" configuration, was initially described in 1985 as a cause of acute neck pain [1]. The syndrome may also be accompanied by neck stiffness, fever, raise of inflammatory indices, chronic neck pain, spinal cord compression or be asymptomatic [2-4]. As the plain radiographs are often clear [5], CT scan is considered as the "cornerstone" for the diagnosis of syndrome [6], which is usually treated with non-steroid anti-inflammatory

\section{Manuscript accepted for publication July 07, 2014}

aThe 2nd Orthopaedic Department, Athens University, Athens, Greece ${ }^{b}$ Orthopaedic Department, Athens University, Athens, Greece

${ }^{\mathrm{c} C}$ Corresponding Author: Vasileios S. Nikolaou, The 2nd Orthopaedic Department, School of Medicine, Athens University, Dimitriou Ralli 21 St, Maroussi, Athens, Greece. Email: vassilios.nikolaou@gmail.com

doi: http://dx.doi.org/10.14740/jmc1837e drugs, colchicine and corticosteroids [1-4, 7-9]. We present a case of a 73-year-old patient who was diagnosed with this rare syndrome, after a fall of a height of approximately $2 \mathrm{~m}$ and cervical spine injury. We also perform a brief review of the literature to shed light onto this rare clinical entity.

\section{Case Report}

A 73-year-old man sustained a cervical spine injury after a fall of approximately $2 \mathrm{~m}$ height. The patient was transported to the local hospital, where the clinical examination showed that he suffered from moderate neck pain with slight disturbance of cervical spine mobility. Neurologically, he was diagnosed with incomplete tetraplegia and was found to have $3 / 5$ strength in his upper extremities, $2 / 5$ strength in his lower extremities, normal sensation and reflexes, normal bladder and bowel function. Plain X-rays of the cervical spine could not clarify the cause of the patient's neurological status (Fig. 1). The patient had been operated 12 years before due to larynx cancer and had a tracheal tube since then. He did not suffer from any other significant comorbidities and had not sustained other injuries from his fall. His Glasgow coma scale was 15/15.

He was transferred to a tertiary spine center for further investigation. A CT scan of the cervical spine was performed,

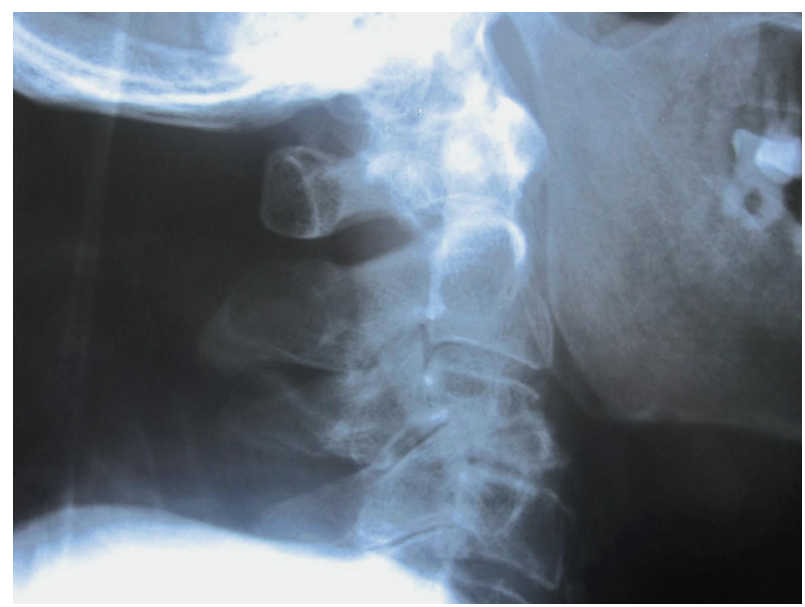

Figure 1. Plain radiographs of the cervical spine revealed only arthritic changes, consistent with the patient's age. 

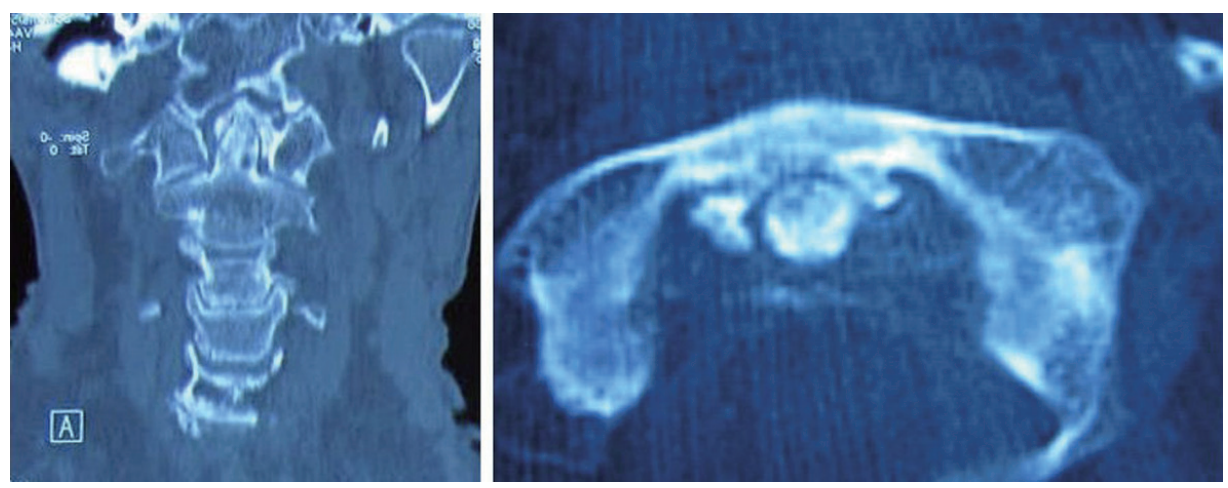

Figure 2. Coronal (left) and axial (right) CT scan of the patient's cervical spine, demonstrating calcification posterolateral to the dens, culminating in a crown-like configuration.

which was negative for fracture or dislocation, but, on the other hand, revealed a periodontoid calcification, indicative of CDS (Fig. 2). The patient denied any previous symptoms of neck pain, stiffness or neurological compromise. His MRI did not show any damage to the spinal cord or soft tissues. He had no fever but his WBC count and CRP were slightly elevated. Since he was admitted to hospital, course of corticosteroids and non-steroid anti-inflammatory medications (NSAIDs) was started and he began to improve neurologically. After a few days he was dismissed with the instruction to use a cervical orthosis for 6 weeks and take common analgesics occasionally. At his follow-up, he was completely clear of signs and symptoms, so the cervical orthosis was discarded. Since then, 4 years after his accident, he has not complained for recurrent pain or functional impairment of his cervical spine.

\section{Discussion}

CDS was first described in 1985 by Bouvet et al, who recognized it as a clinical condition characterized by acute neck pain, accompanied by calcification around the dens of $\mathrm{C} 2$, due to the deposition of crystals of hydroxyapatite or calcium pyrophosphate [1]. The syndrome is more common in the elderly, particularly women after the age of 60 [6]. Its clinical presentation includes acute neck pain with concomitant neck stiffness and possibly fever, while the inflammatory markers are often elevated $[2,3]$. It may be found in up to $5 \%$ of adults over the age of 70 who present to hospital due to neck pain [5]. The syndrome may not often give clinical manifestations, or may cause chronic neck pain or even spinal cord compression [2-4]. Also, it has been reported that calcium pyrophosphate dihydrate crystal deposition in and around atlantoaxial joint may cause fractures of the odontoid process [10].

The differential diagnosis of the syndrome includes mainly meningitis, polymyalgia rheumatica, diskitis, tumor, abscess and giant cell arteritis [11]. Interestingly, the majority of patients with CDS have suffered from symptoms of pseudogout in other joints [5, 12], a fact that supports that most commonly the syndrome is caused by calcium pyrophosphate deposition disease [11]. The etiology of this deposition is unknown, but it seems to be associated with increased levels of pyrophosphate in joints due to increased breakdown of adenosine triphosphate, while also genetic factors (gene ANKH) seem to be involved [8]. However, other authors presented case series in which there is no correlation of CDS and crystal deposition in other joints [13]. In CDS, periodontoid calcification, which is the principal finding in diagnostic imaging, is believed to be caused by deposition of crystals in the fibers of transverse ligament of the atlas [1]. Nevertheless, there have been reported cases where such calcifications are noted in patients with neck pain that suffered from systemic diseases, such as seronegative spondyloarthropathy, systemic sclerosis and rheumatoid arthritis [14]. Furthermore, high calcium levels in the blood, as in hemochromatosis and hyperparathyroidism, are thought to predispose to the syndrome [15].

The gold standard for the diagnosis of the CDS is CT scan [6], because usually plain radiographs fail to highlight the periodontoid calcifications [5]. The CT findings persist for about 3 months after the relief of symptoms of the syndrome [6].

The prognosis of CDS is generally considered as good, but in the literature there is lack of long-term studies of the natural history of the disease and is unknown whether the patient experiences neurologic impairment more frequently with the progress of time [11].

The treatment principally includes the use of NSAIDs and colchicine, which usually leads to a rapid clinical recovery [1$4,7,8]$, although in some cases NSAIDs did not cause clinical improvement and, on the other hand, corticosteroids were proven effective $[7,9]$. In the literature there are cases where spinal cord compression, atlantoaxial instability and myelopathy due to periodontoid calcium pyrophosphate deposits were treated by cervical decompression $[8,16,17]$.

Our case is characterized by the fact that the patient presented an incomplete quadriplegia after a cervical spine injury, but the only finding after the diagnostic imaging was a periodontoid calcification, indicative of CDS. In the literature, we found only one case report, in which this syndrome led to quadriplegia (after clivus destruction) and the patient sustained a posterior surgical decompression and spinal fusion, without, nevertheless, neurological recovery [15]. Up to date, it is not clear if CDS, which was diagnosed incidentally during the investigation of cervical spine trauma, is associated with the neurological status of the patient. 


\section{Conclusion}

CDS is a clinical entity that includes neck pain with possible stiffness, fever and raise of inflammatory markers [2, 3], but it may also be asymptomatic or accompanied by spinal cord compression [2-4]. Our patient's temporary incomplete quadriplegia, not explained by the investigation that was performed, in combination with the incidental finding of CDS, compose an interesting case, characterized by its rarity and difficulty in interpretation.

\section{Author Contributions}

Vasileios Nikolaou and Demetrios Chytas contributed equally to this work and performed the literature research and wrote the paper. Demetrios Korres provided the pictures and the history of the case example and Nicolas Efstathopoulos did the final proof reading of the paper and made final corrections.

\section{References}

1. Bouvet JP, le Parc JM, Michalski B, Benlahrache C, Auquier L. Acute neck pain due to calcifications surrounding the odontoid process: the crowned dens syndrome. Arthritis Rheum. 1985;28(12):1417-1420.

2. Aouba A, Vuillemin-Bodaghi V, Mutschler C, De Bandt M. Crowned dens syndrome misdiagnosed as polymyalgia rheumatica, giant cell arteritis, meningitis or spondylitis: an analysis of eight cases. Rheumatology (Oxford). 2004;43(12):1508-1512.

3. Constantin A, Bouteiller G. Acute neck pain and fever as the first manifestation of chondrocalcinosis with calcification of the transverse ligament of the atlas. Five case-reports with a literature review. Rev Rhum Engl Ed. 1998;65(10):583-585.

4. Wu DW, Reginato AJ, Torriani M, Robinson DR, Reginato AM. The crowned dens syndrome as a cause of neck pain: report of two new cases and review of the literature. Arthritis Rheum. 2005;53(1):133-137.

5. Goto S, Umehara J, Aizawa T, Kokubun S. Crowned Dens syndrome. J Bone Joint Surg Am. 2007;89(12):27322736.

6. Siau K, Lee M, Laversuch CJ. Acute pseudogout of the neck--the crowned dens syndrome: 2 case reports and review of the literature. Rheumatol Int. 2011;31(1):85-88.

7. Sato Y, Yasuda T, Konno S, Kuwayama A, Komatsu K. Pseudogout showing meningoencephalitic symptoms: crowned dens syndrome. Intern Med. 2004;43(9):865868.

8. Baysal T, Baysal O, Kutlu R, Karaman I, Mizrak B. The crowned dens syndrome: a rare form of calcium pyrophosphate deposition disease. Eur Radiol. 2000;10(6):10031005.

9. Takahashi T, Minakata Y, Tamura M, Takasu T, Murakami M. A rare case of crowned dens syndrome mimicking aseptic meningitis. Case Rep Neurol. 2013;5(1):40-46.

10. Kakitsubata Y, Boutin RD, Theodorou DJ, Kerr RM, Steinbach LS, Chan KK, Pathria MN, et al. Calcium pyrophosphate dihydrate crystal deposition in and around the atlantoaxial joint: association with type 2 odontoid fractures in nine patients. Radiology. 2000;216(1):213219.

11. Uh M, Dewar C, Spouge D, Blocka K. Crowned dens syndrome: a rare cause of acute neck pain. Clin Rheumatol. 2013;32(5):711-714.

12. Roverano S, Ortiz AC, Ceccato F, Paira SO. Calcification of the transverse ligament of the atlas in chondrocalcinosis. J Clin Rheumatol. 2010;16(1):7-9.

13. Sekijima Y, Yoshida T, Ikeda S. CPPD crystal deposition disease of the cervical spine: a common cause of acute neck pain encountered in the neurology department. J Neurol Sci. 2010;296(1-2):79-82.

14. Scutellari PN, Galeotti R, Leprotti S, Ridolfi M, Franciosi $\mathrm{R}$, Antinolfi G. The crowned dens syndrome. Evaluation with CT imaging. Radiol Med. 2007;112(2):195-207.

15. Ali S, Hoch M, Dadhania V, Khurana JS. CPPD crowned dens syndrome with clivus destruction: a case report. J Radiol Case Rep. 2011;5(8):30-37.

16. Doita M, Shimomura T, Maeno K, Nishida K, Fujioka H, Kurosaka M. Calcium pyrophosphate dihydrate deposition in the transverse ligament of the atlas: an unusual cause of cervical myelopathy. Skeletal Radiol. 2007;36(7):699-702.

17. Sethi KS, Garg A, Sharma MC, Ahmad FU, Sharma BS. Cervicomedullary compression secondary to massive calcium pyrophosphate crystal deposition in the atlantoaxial joint with intradural extension and vertebral artery encasement. Surg Neurol. 2007;67(2):200-203. 